8-10 days after delivery in 1957 and 36 per cent 11 days or more.

Children, aged 5-9 years, had the shortest average stay in hospital (11 days) and patients, aged 75 and over, the longest (45 days for men and 63 for women). Though this is mainly due to more chronic illness in older people, small children and older people stayed longer than older children and young adults for treatment of the same condition.

Of the patients aged 65 and more, women usually stayed longer than men; for circulatory diseases the average stay for men was 53 days and for women 72 , and for injuries other than head injuries 29 days for men and 41 days for women.

In 'acute' non-teaching hospitals the average length of stay was shortest in medium-sized hospitals. The same was true at all ages and for both sexes, and for many specific diagnoses.
From data on the duration of stay, the number of hospital beds required by different groups of patients can be calculated. The number of beds required per 10,000 population increases greatly with the patient's age, ranging from 20.8 at ages $15-24$ to 188.88 at ages 75 and more for men, and from 18.7 at ages 5-14 to 187.3 at ages 75 and more for women.

Of the 40.8 beds per 10,000 population used for male cases, 13 per cent were taken up by cases of respiratory tuberculosis ; 7 per cent were occupied in treatment of malignant neoplasms, 13 per cent for cardiovascular diseases, 10 per cent for respiratory diseases and 11 per cent for digestive diseases. For females, 48.9 beds per 10,000 population were in use; 7 per cent of them for respiratory tuberculosis, 6 per cent for malignant neoplasms, 8 per cent for digestive diseases and 15 per cent for maternity cases.

\title{
RESPONSE TO HOT ENVIRONMENTS
}

\begin{abstract}
A REPORT* prepared by the Medical Research Council owes its origin to the concern felt by the Board of Admiralty regarding the thermal conditions to which men were exposed during the Second World War when serving in His Majesty's ships in tropical waters. This lod, in 1944, to the appointment of a committee to inquire into and advise on the problems in this field. It was recognized from the outset that investigations would eventually have to be made in a tropical climate, and, in 1948, the Royal Naval Tropical Research Unit was established in Singapore under the joint auspices of the Admiralty and the Medical Research Council.
\end{abstract}

Before the establishment of the unit at Singapore, however, investigations were initiated in London at the National Hospital for Nervous Diseases. In the experiments carried out in London, the physiological effect on men wearing different types of clothing of exposure to high air temperatures was examined at different humidities and air speeds, both when the men were resting and when they were working. On the results of these experiments, for which the subjects had been artificially 'maximally acclimatized' by previous exposure to hot conditions in the laboratory, it was possible to base tentative recommendations concerning the conditions of warmth that were acceptable in ships at sea in tropical waters. The results also seemed to indicate that the "effective temperature scale', which had been accepted for use as a measure of the severity of environment in ships, was misleading in certain respects. This led Dr. B. McArdle to devise a new index, the 'predicted four-hour sweat-rate' (P4SR), for the assessment of hot environments.

With the establishment of the unit at Singapore similar investigations were carried out. These confirmed largely the previous findings in London and, though they did not entirely support the criticisms of the 'effective temperature scale', they did demonstrate that the 'predicted four-hour sweat-rate' was

* Privy Council. Medical Research Council Report Series, No. 298. Physiological Responses to Hot Environments. Compiled by $\dot{\mathbf{R}} . \mathbf{K}$. Macpherson. (An account of work done in Singapore, 1948-1953, at the Royal Naval Tropical Research Unit, with an appendix on preliminary work done at the National Hospital for Nervous Diseases,
London.) $P p$. $x v+323$. (London: H.M. Stationery Office, 1960.) 35s. net. an accurate and convenient measure of the severity of indoor hot-environments. The work at Singapore was, however, extended to compare the relative tolerance to heat of men who had been artificially 'maximally acclimatized' in the laboratory, with those who had been 'naturally acclimatized' by residence in a tropical climate. Investigations were also made of the stress imposed by radiant heat of long wavelength and by different rates of energy expenditure. Work carried out to determine the upper limits of warmth to be permitted in ships was extended to determine the upper limits that could be physically tolerated and the position of the 'comfort' zone. In addition, a number of physiological problems associated with these main lines of research was examined.

Now that the results of all the investigations in London and in Singapore have been brought together, it is clear that considerable additions to knowledge have been achieved. Much information has been obtained on the relative effects of various levels of air temperature, humidity, air speed, and radiant heat, and about the limits of heat which people can tolerate.

Answers have been provided to some of the practical problems of the Navy ; the critical examination of a widely used scale of environmental heat-stress and the production and testing of a new index have improved and added to the techniques available for evaluating the severity of different climatic environments. As well as achieving these practical ends, this work has extended knowledge of the physiological mechanisms involved in the process of adaptation to hot environments and has indicated the direction for future research.

The report has been compiled by Dr. R. K. Macpherson with the assistanc $\rightarrow$ of an editorial board composed of Dr. T. Bedford, Dr. O. G. Edholm, Surgeon Captain F. N. Ellis, and Dr. N. H. Mackworth. It brings together much patient and arduous work by a number of service-mon and civilians, and it is a fine example of how long-term investigations should be planned, organized and controlled. Many of its findings should be of interest to industrial organizations where hot processes are involved; managements in the new Indian steelworks should find much of value. 